\title{
Effects of regularisation priors on dynamic PET Data
}

\author{
Liliana Caldeira*, Juergen Scheins, Nuno da Silva, Michaela Gaens, N Jon Shah \\ From PSMR14: 3rd Conference in PET/MR and SPECT/MR \\ Kos Island, Greece. 19-21 May 2014
}

Institute of Neuroscience and Medicine-4, Forschungszentrum Juelich, Germany
Dynamic PET provides temporal information about tracer uptake. However, each PET frame has usually low statistics, resulting in noisy images. The goal is to study effects of prior regularisation on dynamic PET data. Quantification and noise in imagedomain and time-domain as well as impact on parametric images is assessed.

Dynamic PET data for the Siemens 3T MR-BrainPET was simulated with time-activity curves (TAC) of [1] F-FDG obtained analytically using realistic values for the kinetic model (Table 1). The total number of true counts was $6 \times 10^{8}$ and scatter and random fractions were both $35 \%$. The data consists of 23 frames as applied in our clinical protocol. For reconstruction, the Ordinary Poisson Ordered Subset Expectation Maximisation (OP-OSEM) method was used in PRESTO [1], which allows to use several 3D priors [2]. The Median Root Prior (MRP) used a 3x3x3 neighbourhood and a Bayes parameter of 0.1 for all frames. Patlak parametric images were calculated using PMOD software.

Figure 1 shows that the MRP OP-OSEM reduces image noise in WM, GM and AIF: from $50 \%$ reduction in low-count frames to $10 \%$ reduction in high-count frames (e.g. Frame 24). Furthermore, Patlak parametric images also look smoother for MRP OPOSEM than for OP-OSEM.

Figure 2 shows similar quantification for both reconstruction methods (with similar RCs). OP-OSEM presents higher RCs than MRP OP-OSEM in high-count frames (up to 9\%), while in low-count frames MRP OP-OSEM presents higher RCs (up to 9\%). This is probably due to the fact that the Bayes parameter is count-dependent.

This study shows improvement on PET image quality in terms of noise (up to 50\% reduction) as well as in parametric images when using prior regularisation in dynamic PET data. Both OP-OSEM and MRP OP-OSEM show similar quantification, with higher RCs for MRP OP-OSEM in low-count frames.

Table 1 Kinetic values used for simulation of two tissues: White-Matter (WM) and Gray-Matter (GM), extracted from real acquired volunteer data.

\begin{tabular}{ccccc}
\hline & $\mathrm{k} 1(\mathrm{~mL} / \mathrm{cc} / \mathrm{min})$ & $\mathrm{k} 2\left(\mathrm{~min}^{-1}\right)$ & $\mathrm{k} 3\left(\mathrm{~min}^{-1}\right)$ & $\mathrm{k} 4\left(\mathrm{~min}^{-1}\right)$ \\
\hline White-Matter $(\mathrm{WM})$ & 0.054 & 0.111 & 0.0045 & 0.0059 \\
\hline Gray-Matter (GM) & 0.103 & 0.133 & 0.063 & 0.0068 \\
\hline
\end{tabular}

\section{SpringerOpen ${ }^{\circ}$}

(C) 2014 Caldeira et al; licensee Springer This is an Open Access article distributed under the terms of the Creative Commons Attribution License (http://creativecommons.org/licenses/by/4.0), which permits unrestricted use, distribution, and reproduction in any medium, provided the original work is properly cited. 


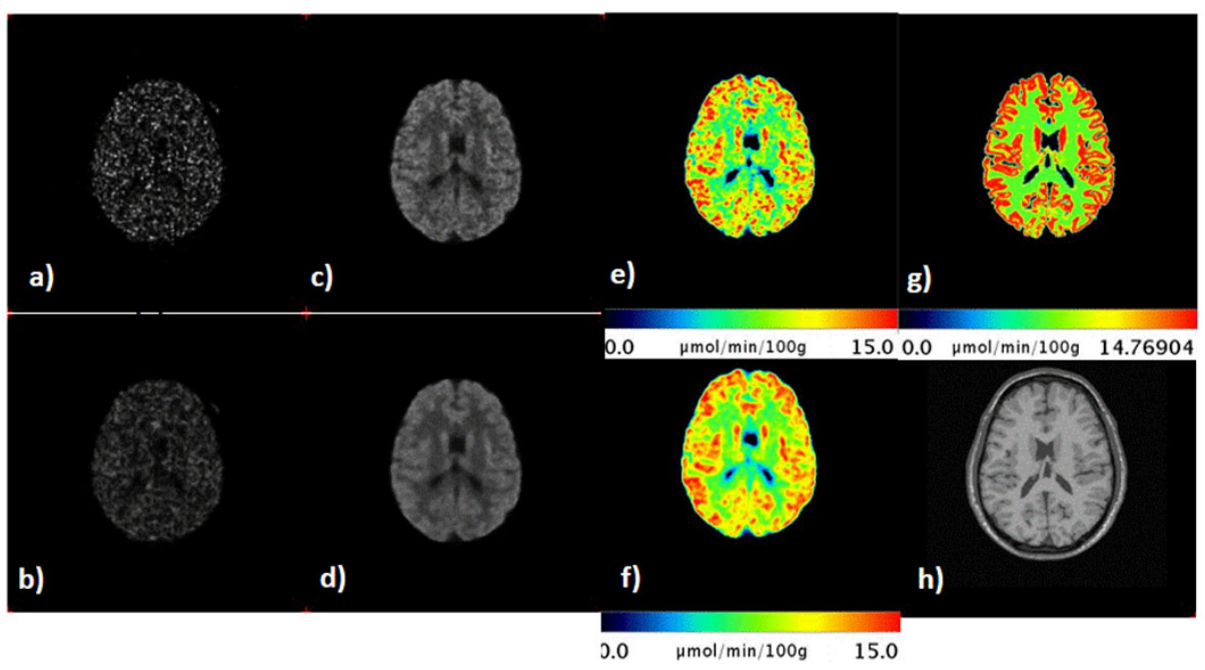

Figure 1 a) Reconstructed images without MRP corresponding to Frame 14 (2.77× $10^{6}$ counts); b) Reconstructed images with MRP corresponding to Frame 14 (2.77× $10^{6}$ counts); c) Reconstructed images

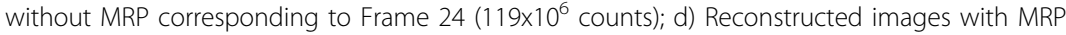

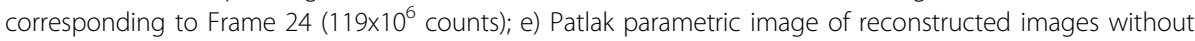
prior; f) Patlak parametric image of reconstructed images with prior; g) Patlak Parametric Images of ground truth; h) MRI image for anatomical reference.
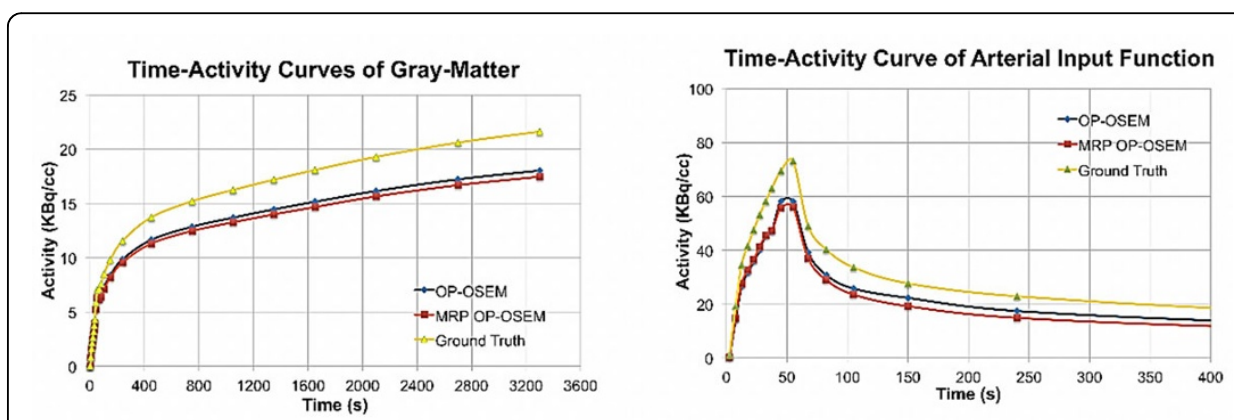

Figure 2 TAC using OP-OSEM reconstruction without and with MRP for two different regions: a) GM and b) AlF.

Published: 29 July 2014

\section{References}

1. Scheins JJ, et al: Fully-3D PET image reconstruction using scanner-independent, adaptive projection data and highly rotation-symmetric voxel assemblies. IEEE TMI 2011, 30(3):879-92.

2. Caldeira $L$, et al: Evaluation of two methods for using MRI information in PET reconstruction. NIMA 2013, 702:29-33.

\section{doi:10.1186/2197-7364-1-S1-A46}

Cite this article as: Caldeira et al:: Effects of regularisation priors on dynamic PET Data. EJNMMI Physics 2014

1(Suppl 1):A46. 